\title{
On the Graph Edit Distance cost: Properties and Applications ${ }^{1}$
}

\author{
Albert Solé-Ribalta ${ }^{2}$, Francesc Serratosa and Alberto Sanfeliu \\ Department of Computer Science and Mathematics \\ Universitat Rovira i Virgili (URV), Avda. Països Catalans, 26, 43007 Tarragona. \\ \{albert.sole@urv.cat\},\{francesc.serratosa@urv.cat\} \\ Institut de Robòotica i Informàtica Industrial CSIC-UPC \\ Barcelona, Spain \\ \{sanfeliu@iri.upc.edu\}
}

\begin{abstract}
We model the edit distance as a function in a labelling space. A labelling space is an Euclidean space where coordinates are the edit costs. Through this model, we define a class of cost. A class of cost is a region in the labelling space that all the edit costs have the same optimal labelling. Moreover, we characterise the distance value through the labelling space. This new point of view of the edit distance gives as the opportunity of defining some interesting properties that are useful for a better understanding of the edit distance. Finally, we show the usefulness of these properties through some applications.
\end{abstract}

Keywords: Graph Edit Distance, Graph Edit Costs, graph similarity, graph distance, errorcorrecting graph isomorphism.

\section{Introduction}

Graphs are of crucial importance in pattern recognition. Graph structures are used to model several kinds of problems in several pattern recognition fields [1] such as general 2D/3D object recognition [2-7], object and scene view alignment [8-11], SLAM [12, 13], and graph prototyping [14-17].

One of the most essential researched problems in the field of pattern recognition in relation to graph structures is the definition of a similarity measure between graphs. This is an important part of the error tolerant graph matching [18] problem. Error tolerant graph matching aims to compute a bijection between nodes of two graphs that minimizes some kind of objective function. The general case of this problem is known to be NP-complete [19]. Generally, the objective function is related to a graph distance. In the ideal case, graph

\footnotetext{
${ }^{1}$ This research is supported by "Consolider Ingenio 2010": project CSD2007-00018, by the CICYT project DPI201017112 and by the Universitat Rovira i Virgili through a PhD research grant.

${ }^{2}$ Corresponding author.
} 
distances should be a metric and thus fulfil the four metric properties: (1) non-negativity, (2) identity of indiscernible, (3) symmetry, and (4) triangle inequality. Some graph distances appear in the literature [20-22], but probably the most well known distance is the Graph Edit Distance [23]. The application of graph edit distance is extensive [24] and therefore numerous algorithms to compute the Graph Edit Distance can be found in the literature, such as [25-29]. Moreover, some theoretical papers describe properties of a particular definition of the Graph Edit Distance [30, 31] and [32].

The use of the Graph Edit Distance tailored to a particular problem requires some application-dependent functions to be defined. The optimal definition and specification of these functions is no trivially undertaken, and several works have addressed to this task. The most relevant of these are $[5,33,34]$ and [35]. The main contribution of these works has been to prove that String Edit Distance and Graph Edit Distance contain several classes of cost. In this article, we define some un-described properties of the Graph Edit Distance. Our specific definition and interpretation of the Graph Edit Distance allows each class of cost to be described using a plane equation and allows the shape of each class of cost to be described as well. The use of these new properties is twofold. On the one hand they can be used to improve performance of existing algorithms and, on the other hand, they can be used to develop more efficient graph algorithms.

An interesting survey summarizing the most important contributions on Graph Edit Distance has recently been published [24].

The aim of this paper is to go a step further about the findings related about edit costs presented by professor Horst Bunke and his colleagues. They presented some new ideas about the existence of classes of edit costs in strings [32] and graphs [30]. They show that the edit costs can be clustered in some classes, in which the edit distance behaves in a similar manner. We present here a new methodology to represent these classes of cost and some properties. We also show the usefulness of this methodology and properties through some applications. 
This article is structured as follows. Section 2 introduces the required definitions for the rest of the article. Section 3 describes several new properties of the Graph Edit Distance and gives some insights into the labellings that the distance can optimize. Section 4 describes some possible uses and applications of the previously described properties. Finally, section 5 highlights the main contributions of the article and draws some conclusions.

\section{Basic definitions and Graph Edit Distance}

\section{Attributed Graph}

Let $\Delta_{v}$ and $\Delta_{e}$ denote the domains of possible values for attributed vertices and arcs, respectively. An attributed graph $A G$ (over $\Delta_{v}$ and $\left.\Delta_{e}\right)$ is defined by a tuple $G=\left(\Sigma_{v}, \Sigma_{\mathrm{e}}, \gamma_{v}, \gamma_{e}\right)$, where $\Sigma_{v}=\left\{v_{k} \mid k=1, \ldots, R\right\}$ is the set of vertices (or nodes), $\Sigma_{e} \in\left\{e_{i j} \mid i, j \in\{1, \ldots, R\}, i<\right.$ $j\}$ is the set of undirected arcs (or edges), $\gamma_{v}: \Sigma_{v} \rightarrow \Delta_{v}$ assigns attribute values to vertices and $\gamma_{e}: \Sigma_{e} \rightarrow \Delta_{e}$ assigns attribute values to arcs.

\section{Error correcting graph isomorphism between graphs}

Let $\mathrm{G}^{\mathrm{p}}=\left(\Sigma_{\mathrm{v}}^{\mathrm{p}}, \Sigma_{\mathrm{e}}^{\mathrm{p}}, \gamma_{\mathrm{v}}^{\mathrm{p}}, \gamma_{\mathrm{e}}^{\mathrm{p}}\right)$ and $\mathrm{G}^{\mathrm{q}}=\left(\Sigma_{\mathrm{v}}^{\mathrm{q}}, \Sigma_{\mathrm{e}}^{\mathrm{q}}, \gamma_{\mathrm{v}}^{\mathrm{q}}, \gamma_{\mathrm{e}}^{\mathrm{q}}\right)$ be two attributed graphs of order $\mathrm{R}^{\mathrm{p}}$ and $\mathrm{R}^{\mathrm{q}}$. To allow maximum flexibility in the matching process, graphs can be extended with null nodes [36] to be of order $\mathrm{R}^{\mathrm{p}}+\mathrm{R}^{\mathrm{q}}$. We will refer to null nodes of $\mathrm{G}^{\mathrm{p}}$ and $\mathrm{G}^{\mathrm{q}}$ by $\widehat{\Sigma}_{\mathrm{v}}^{\mathrm{p}} \subseteq \Sigma_{\mathrm{v}}^{\mathrm{p}}$ and $\widehat{\Sigma}_{\mathrm{v}}^{\mathrm{q}} \subseteq \Sigma_{\mathrm{v}}^{\mathrm{q}}$ respectively. Let $\mathrm{T}$ be a set of all possible bijections between two vertex sets $\Sigma_{\mathrm{v}}^{\mathrm{p}}$ and $\Sigma_{\mathrm{v}}^{\mathrm{q}}$. Bijection $\mathrm{f}^{\mathrm{p}, \mathrm{q}}: \Sigma_{\mathrm{v}}^{\mathrm{p}} \rightarrow \Sigma_{\mathrm{v}}^{\mathrm{q}}$, assigns every vertex of $\mathrm{G}^{\mathrm{p}}$ to only one vertex of $\mathrm{G}^{\mathrm{q}}$. The bijection between arcs, denoted by $\mathrm{f}_{\mathrm{e}}^{\mathrm{p}, \mathrm{q}}$, is defined accordingly to the bijection of their terminal nodes. In other words:

$$
\begin{gathered}
f_{e}^{p, q}\left(e_{a b}^{p}\right)=e_{i j}^{q} \Rightarrow f^{p, q}\left(v_{a}^{p}\right)=v_{i}^{q} \wedge f^{p, q}\left(v_{b}^{p}\right)=v_{j}^{q} \\
v_{a}^{p}, v_{b}^{p} \in \Sigma_{\mathrm{v}}^{\mathrm{p}}-\hat{\Sigma}_{\mathrm{v}}^{\mathrm{p}} \text { and } v_{i}^{q}, v_{j}^{q} \in \Sigma_{\mathrm{v}}^{\mathrm{q}}-\hat{\Sigma}_{\mathrm{v}}^{\mathrm{q}}
\end{gathered}
$$

We define non-existent or null edges by $\widehat{\Sigma}_{\mathrm{e}}^{\mathrm{p}} \subseteq \Sigma_{\mathrm{e}}^{\mathrm{p}}$ and $\widehat{\Sigma}_{\mathrm{e}}^{\mathrm{q}} \subseteq \Sigma_{\mathrm{e}}^{\mathrm{q}}$. 


\section{Graph Edit Distance between two graphs}

One of the most widely used methods to evaluate an error correcting graph isomorphism is the Graph Edit Distance [23]. The basic idea behind the Graph Edit Distance is to define a dissimilarity measure between two graphs. This dissimilarity is defined as the minimum amount of distortion required to transform one graph into the other. To this end, a number of distortion or edit operations, consisting of insertion, deletion and substitution of both nodes and edges are defined. Then, for every pair of graphs $\left(G^{p}\right.$ and $\left.G^{q}\right)$, there is a sequence of edit operations, or an edit path editPath $\left(G^{p}, G^{q}\right)=\left(\varepsilon_{1}, \ldots, \varepsilon_{k}\right)$ (where each $\varepsilon_{i}$ denotes an edit operation) that transforms one graph into the other. In general, several edit paths may exist between two given graphs. This set of edit paths is denoted by $\vartheta$. To quantitatively evaluate which edit path is the best, edit cost functions are introduced. The basic idea is to assign a penalty cost to each edit operation according to the amount of distortion that it introduces in the transformation.

Each editPath $\left(G^{p}, G^{q}\right) \in \vartheta$ can be related to a univocal graph isomorphism $f^{p, q} \in T$ between the involved graphs. In this way, each edit operation assigns a node of the first graph to a node of the second graph. Deletion and insertion operations are transformed to assignations of a non-null node of the first or second graph to a null node of the second and first graph. Substitutions simply indicate node-to-node assignations. Using this transformation, given two graphs, $G^{p}$ and $G^{q}$, and a bijection between their nodes, $f^{p, q}$, the Graph Edit cost is given by (Definition 7 of [30]): 
$\operatorname{Edit} \operatorname{Cost}\left(G^{p}, G^{q}, f^{p, q}\right)$

$$
\begin{aligned}
& =\sum_{\substack{v_{a}^{p} \in \Sigma_{v}^{p}-\widehat{\Sigma}_{v}^{p} \\
v_{i}^{q} \in \Sigma_{v}^{q}-\mathbb{\Sigma}_{v}^{q}}} C_{n s}\left(v_{a}^{p}, v_{i}^{q}\right)+\sum_{\substack{v_{a}^{p} \in \in_{v}^{p}-\widehat{\Sigma}_{v}^{p} \\
v_{i}^{q} \in \bar{\Sigma}_{v}^{q}}} C_{n d}\left(v_{a}^{p}, v_{i}^{q}\right)+\sum_{\substack{v_{a}^{p} \in \overleftarrow{E}_{v}^{p} \\
v_{i}^{q} \in \Sigma_{v}^{q}-\widehat{\Sigma}_{v}^{q}}} C_{n i}\left(v_{a}^{p}, v_{i}^{q}\right) \\
& +\sum_{\substack{e_{a b}^{p} \in \Sigma_{e}^{p}-\widehat{\Sigma}_{e}^{p} \\
e_{i j}^{q} \in \Sigma_{e}^{q}-\widehat{\Sigma}_{e}^{q}}} C_{e s}\left(e_{a b}^{p}, e_{i j}^{q}\right)+\sum_{\substack{e_{a b}^{p} \in \Sigma_{e}^{p}-\hat{\Sigma}_{e}^{p} \\
e_{i j}^{q} \widehat{\Sigma}_{e}^{q}}} C_{e d}\left(e_{a b}^{p}, e_{i j}^{q}\right)+\sum_{\begin{array}{c}
e_{a b}^{p} \in \tilde{\Sigma}_{e}^{p} \\
e_{i j}^{q} \in \Sigma_{e}^{q}-\widehat{\Sigma}_{e}^{q}
\end{array}} C_{e i}\left(e_{a b}^{p}, e_{i j}^{q}\right) \\
& f^{p, q}\left(v_{a}^{p}\right)=v_{i}^{q} \text { and } f_{e}^{p, q}\left(e_{a i}^{p}\right)=e_{i j}^{q}
\end{aligned}
$$

where $C_{n s}$ is the cost of substituting node $v_{a}^{p}$ of the $G^{p}$ for node $f^{p, q}\left(v_{a}^{p}\right)$ of $G^{q}, C_{n d}$ is the cost of deleting node $v_{a}^{p}$ of $G^{p}$ and $C_{n i}$ is the cost of inserting node $v_{i}^{q}$ of $G^{q}$. And for edges: $C_{e s}$ is the cost of substituting edge $e_{a b}^{p}$ of graph $G^{p}$ for edge $f_{e}^{p, q}\left(e_{a b}^{p}\right)$ of $G^{q}, C_{e d}$ is the cost of assigning edge $e_{a b}^{p}$ of $G^{p}$ to a non-existing edge of $G^{q}$ and $C_{e i}$ is the cost of assigning edge $e_{a b}^{q}$ of $G^{q}$ to a non-existing edge of $G^{p}$.

Finally, the Graph Edit Distance is defined as the minimum cost under any bijection in $T$ :

$$
\operatorname{EditDistance}\left(G^{p}, G^{q}\right)={ }_{f^{p, q} \in T}^{\operatorname{argmin}} \operatorname{EditCost}\left(G^{p}, G^{q}, f^{p, q}\right)
$$

Using this definition, Graph Edit Distance depends essentially on $C_{v s}, C_{v d}, C_{v i}, C_{e s}, C_{e d}$ and $C_{e i}$ functions. Several definitions of these functions exist. We focus first on the definition of the functions $C_{n s}$ and $C_{e s}$. The most common approaches are the following. The first and simplest approach considers cost $C_{n s} \in\left\{0, K_{n s}\right\}$ where $C_{n s}\left(v_{a}^{p}, v_{i}^{q}\right)=K_{n s}$ if $d\left(\gamma_{v}^{p}\left(v_{a}^{p}\right), \gamma_{v}^{q}\left(v_{i}^{q}\right)\right)<$ Threshold otherwise $C_{n s}=0, d$ is defined as a distance function over the domain of the attributes. A specific example of this cost can be found in fingerprint verification [37] where $C_{n s} \in\{0,1\}$ or in [30,31]. The second and most frequently used approach corresponds to the case in which $C_{n s}\left(v_{a}^{p}, v_{b}^{q}, \theta_{v}\right) \in \mathbb{R}$, and node substitution cost depends on the attributes of the nodes and possibly on some other parameters $\theta_{v}$, as shown in [34],[26] and [8], among others. Similar approaches can be used to define $C_{e s}$. With regard to $C_{n d}, C_{n i}, C_{e d}$ and $C_{e i}$, these functions usually simply assign a constant cost. However, they can also depend on node or edge attributes [36] [38, 39]. 
Nodes and edges can be mapped, by functions $\gamma_{v}^{p}$ and $\gamma_{e}^{p}$, to several types of data: nominal, ordinal or modulo. Depending on the data type a particular distance function is required (see section 3 of [40]).

Several specific joint definitions for $C_{n s}, C_{n d}, C_{n i}, C_{e s}, C_{e d}$ and $C_{e i}$ functions have been theoretically studied. We highlight [31] and [30] which are described in Table 1.

\begin{tabular}{|l|c|c|c|c|c|c|}
\hline Reference & $C_{n s}$ & $C_{n d}$ & $C_{n i}$ & $C_{e s}$ & $C_{e d}$ & $C_{e i}$ \\
\hline$[31],[30]$ & $\{0, \infty\}$ & $\{1\}$ & $\{1\}$ & $\{0, \infty\}$ & $\{0\}$ & $\{0\}$ \\
\hline$[30]$ & $d\left(v_{a}^{p}, v_{i}^{q}, \theta_{v}\right) \in \mathbb{R}$ & $\left\{K_{n d}\right\}$ & $\left\{K_{n i}\right\}$ & $d\left(e_{a b}^{p}, v_{i j}^{q}, \theta_{e}\right) \in \mathbb{R}$ & $\{0\}$ & $\{0\}$ \\
\hline
\end{tabular}

Table 1: graph edit cost defined in [31] and [30].

The specific cases studied in [30] and [31] yield to several interesting properties. The costs of first row of Table 1 relate the Graph Edit Distance with the maximal common sub-graph. In this way computing the graph edit distance with this specific costs leads to the computation of the maximal common sub-graph. The cost given in the second row has been studied in [30]. Note that the cost of inserting and deleting an edge is always zero. In the definition of [30] authors assume that the graphs are complete graphs and a non-existing edge is an edge with "null" label. In this case the cost of deleting and inserting and edge can be encoded in the edge substitution cost. With this definition authors describe several classes of costs that optimize at the same final labelling. In this article, we aim to follow the same direction and give a deepest characterization of these classes of costs. To this aim we slightly modify the graph edit definition of Table 1 second row. This new definition is given in Table 2.

\begin{tabular}{|c|c|c|c|c|c|}
\hline$C_{n s}$ & $C_{n d}$ & $C_{n i}$ & $C_{e s}$ & $C_{e d}$ & $C_{e i}$ \\
\hline$d\left(v_{a}^{p}, v_{i}^{q}, \theta_{v}\right) \in \mathbb{R}$ & $K_{n}$ & $K_{n}$ & $d\left(e_{a b}^{p}, v_{i j}^{q}, \theta_{e}\right) \in \mathbb{R}$ & $K_{e}$ & $K_{e}$ \\
\hline
\end{tabular}

Table 2: particularization of Graph Edit Distance.

Note that we are able to codify the same information, however edge insertion and edge deletions are considered in a separate cost function. Besides, we impose the requirement that $C_{n d}(v)=C_{n i}(v)=K_{n}, \forall v$ and $C_{e d}(e)=C_{e i}(e)=K_{e}, \forall e$. This requirement is necessary for 
our development and moreover for the Graph Edit Distance to fulfil the symmetric property of a metric.

\section{Class of Costs and Edit Surface}

A Labelling Space is an Euclidean space where the coordinates are the edit costs. Given a pair of graphs, we can select some regions in this space such that all points in the labelling space obtain the same optimal labelling (the labelling that obtains the edit distance). We call each region as Class of Costs. Moreover, given the labelling space and two graphs, we can define a function defined over all the labelling space that its value in each point is the distance value between both graphs and given some specific edit costs. We call this function Edit Surface.

In this section, we first give some basic definitions and then we present two properties of the Class of Costs and one property of the Edit Surface. From now to the rest of the paper, we use the particular specification of the Graph Edit Distance given in Table 2. Therefore, the labelling space is a bi-dimensional space with the axis $k_{n}$ and $k_{e}$.

\subsection{Basic Definitions}

Definition 1: Edit Cost

Given two graphs, $G^{p}$ and $G^{q}$, an isomorphism $f \in T$ between them and two constant values $\left(K_{n}, K_{e}\right) \in \mathbb{R}^{2^{+}}$, the graph edit cost is given by:

$$
\operatorname{EditCost}_{K_{n}, K_{e}}\left(G^{p}, G^{q}, f\right)=a_{f} K_{n}+b_{f} K_{e}+c_{f}
$$

$a_{f}$ refers to the number of inserted and deleted nodes and can be computed as:

$$
a_{f}=\sum_{\left\{\forall v_{a}^{p} \in \Sigma_{v}^{p}-\widehat{\Sigma}_{v}^{p}\right\}\left\{\forall v_{i}^{q} \in \widehat{\Sigma}_{v}^{q}\right\}} H_{v}\left(v_{a}^{p}, v_{i}^{q}\right)+\sum_{\left\{\forall v_{a}^{p} \in \widehat{\Sigma}_{v}^{p}\right\}\left\{\forall v_{i}^{q} \in \Sigma_{v}^{q}-\widehat{\Sigma}_{v}^{q}\right\}} H_{v}\left(v_{a}^{p}, v_{i}^{q}\right)
$$

$b_{f}$ refers to the number of inserted and deleted edges and can be computed as:

$$
b_{f}=\sum_{\left\{\forall e_{a b}^{p} \in \Sigma_{e}^{p}-\widehat{\Sigma}_{e}^{p}\right\}} \sum_{\left\{\forall e_{i j}^{q} \in \widehat{\Sigma}_{e}^{q}\right\}} H_{e}\left(e_{a b}^{p}, e_{i j}^{q}\right)+\sum_{\left\{\forall e_{a b}^{p} \in \widehat{\Sigma}_{e}^{p}\right\}\left\{\forall e_{i j}^{q} \in \Sigma_{e}^{q}-\widehat{\Sigma}_{e}^{q}\right\}} H_{e}\left(e_{a b}^{p}, e_{i j}^{q}\right)
$$

$c_{f}$ refers to the cost of substituting nodes and edges, this last cost can be computed as: 


$$
\begin{aligned}
c_{f}= & \sum_{\left\{\forall v_{a}^{p} \in \Sigma_{v}^{p}-\widehat{\Sigma}_{v}^{p}\right\}\left\{\forall v_{i}^{q} \in \Sigma_{v}^{q}-\widehat{\Sigma}_{v}^{q}\right\}} H_{v}\left(v_{a}^{p}, v_{i}^{q}\right) * d\left(v_{a}^{p}, v_{i}^{q}, \theta_{v}\right) \\
& +\sum_{\left\{\forall e_{a b}^{p} \in \Sigma_{e}^{p}-\widehat{\Sigma}_{e}^{p}\right\}} \sum_{\left\{\forall e_{i j}^{q} \in \Sigma_{e}^{q}-\widehat{\Sigma}_{e}^{q}\right\}} H_{e}\left(e_{a b}^{p}, e_{i j}^{q}\right) * d\left(e_{a b}^{p}, e_{i j}^{q}, \theta_{e}\right)
\end{aligned}
$$

$H_{v}\left(v_{a}^{p}, v_{i}^{q}\right)$ and $H_{e}\left(e_{a b}^{p}, e_{i j}^{q}\right)$ are computed as:

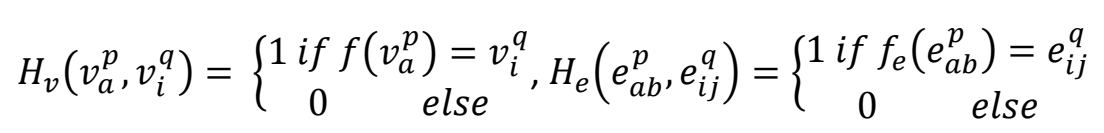

Using this particular definition, the Graph Edit Cost function can be represented in a 3dimensional space where $\mathrm{x}$-axis corresponds to $K_{n}, \mathrm{y}$-axis corresponds to $K_{e}$ and z-axis corresponds to EditCost. Note that EditCost depends linearly on $K_{n}$ and $K_{e}$.

\section{Definition 2: Edit Distance}

Given two graphs $G^{p}$ and $G^{q}$ and two constant values $\left(K_{n}, K_{e}\right) \in \mathbb{R}^{2^{+}}$, the graph edit distance is defined as:

$$
\begin{gathered}
\text { EditDistance }_{K_{n}, K_{e}}\left(G^{p}, G^{q}\right)=\min _{f \in T}\left\{\operatorname{EditCost}_{K_{n}, K_{e}}\left(G^{p}, G^{q}, f\right)\right\} \\
=\min _{f \in T}\left\{a_{f} K_{n}+b_{f} K_{e}+c_{f}\right\}
\end{gathered}
$$

In other words, the Graph Edit Distance is the minimum cost that can be obtained for concrete values of $K_{n}$ and $K_{e}$.

\section{Definition 3: Class of Cost}

Given two graphs, $G^{p}$ and $G^{q}$, and an isomorphism $f \in T$ between them, a class of cost $\Omega^{p, q}(f)$ is the sub-set of values in $\mathbb{R}^{2^{+}}$in which $f$ is the isomorphism whereby the minimum graph edit cost is obtained,

$$
\Omega^{p, q}(f)=\left\{\left(K_{n}, K_{e}\right) \in \mathbb{R}^{2^{+}} \mid f=\operatorname{argmin}_{f, \in T}\left\{a_{f} K_{n}+b_{f} K_{e}+c_{f}\right\}\right\}
$$

We write $\Omega(f)$ instead of $\Omega^{p, q}(f)$ when no confusion is possible. We denominate the set of all classes of cost given to graphs $G^{p}$ and $G^{q}$ by $\widehat{\Omega}^{p, q}$. 


\section{Definition 4: Edit Surface}

Given two graphs, $G^{p}$ and $G^{q}$, and a bi-dimensional space composed of values $\left(K_{n}, K_{e}\right)$ in $\mathbb{R}^{2^{+}}$, we define the Edit Surface as,

$$
\begin{gathered}
\text { EditSurface }_{G^{p}, G^{p}}:_{\mathbb{R}^{2^{+}} \rightarrow \mathbb{R}^{+},} \\
{\text {EditSurface }{ }_{G}{ }^{p}, G^{q}}\left(K_{n}, K_{e}\right)=\text { EditDistance }_{K_{n}, K_{e}}\left(G^{p}, G^{q}\right)=\min _{f \in T}\left\{a_{f} K_{n}+b_{f} K_{e}+c_{f}\right\}
\end{gathered}
$$

\subsection{Properties of the Class of Costs}

Property 1. Given two graphs, $G^{p}$ and $G^{q}$, any class of cost $\Omega(f)$ is either empty or its values form a convex polygon in the bi-dimensional space composed of $\left(K_{n}, K_{e}\right) \in \mathbb{R}^{2^{+}}$.

\section{Demonstration}

Given two graphs, $G^{p}$ and $G^{q}$, and the labelling $f$. We see that for $f$ to yield the Graph Edit Distance at a concrete point $\left(K_{n}, K_{e}\right)$, its cost must be less than or equal to the cost which can be obtained with any other labelling $f^{\prime} \in T$. That is, the following system of inequalities must hold:

$$
a_{f} K_{n}+b_{f} K_{e}+c_{f} \leq a_{f^{\prime}} K_{n}+b_{f^{\prime}} K_{e}+c_{f^{\prime}}, \forall f^{\prime} \in(T-f)
$$

Each of the above inequalities (each $f^{\prime}$ ) divides $\mathbb{R}^{2+}$ into two parts by means of a linear equation. It is known that the intersection of any set of linear inequalities is a convex polygon [41]. Consequently, each optimal labelling appears only in a single convex polygon $\square$

Property 2. Given two graphs, $G^{p}$ and $G^{q}$, and a class of cost $\Omega^{p, q}(f)$, any class of cost $\Omega^{p, q}\left(f^{\prime}\right)$ where $f^{\prime} \in(T-f), a_{f}=a_{f \prime}, b_{f}=b_{f}$, and $c_{f}=c_{f}$ is optimal at the same set of points as $\Omega(f)$.

\section{Demonstration}

This property is easily deduced through equation (12) $\square$ 


\section{Discussion of property 1 and 2}

Using Property 1 , we see that $\Omega(f), \forall f$ optimal, divides $\mathbb{R}^{2+}$ into convex polygons, where each polygon defines a class of cost $\Omega(f)$. A class forms a convex polygon with finite area if its values of $K_{n}$ and $K_{e}$ are finite. Otherwise, the area is infinite.

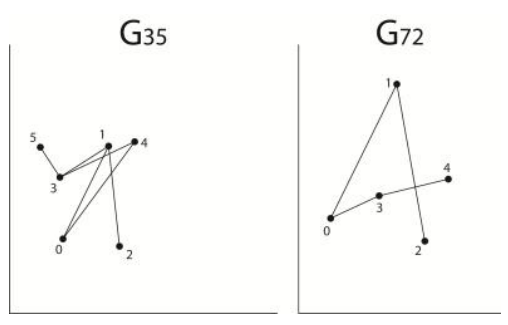

Figure 1.a: two graphs.

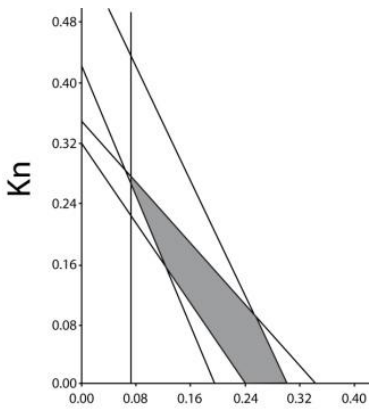

$\mathrm{Ke}$

Figure 1.b: example of a

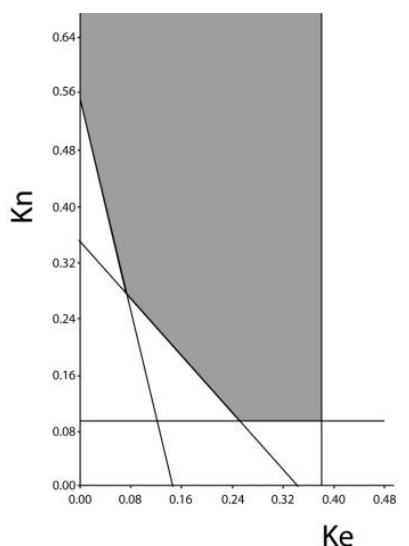

Figure 1.c: example of infinite area.

Fig. 1.b and 1.c show an example of Property 1. Fig. 1.a shows two graphs of the well known graph dataset [42]. Examples correspond to graph 35 and 72 of class A. Fig. 1.b and 1.c show how two labellings are described by the intersection of a set of inequalities, each line corresponding to a concrete inequality of (12). Fig. 1.b shows a finite polygon and Fig. 1.c shows an infinite area of a class.

Note that the above formulation allows dividing $\mathbb{R}^{2^{+}}$into convex polygons, each of which corresponds to an optimal labelling. Note also that it is possible for (12) to produce an empty intersection, in which case the tested labelling $f$ is never optimal at any $\left(K_{n}, K_{e}\right)$.

Knowing that the labelling space is tessellated with labellings it is interesting to see how these labellings tend to be distributed and their relation to the values and meaning of $K_{n}$ and $K_{e}$, specially for the extreme values of $\left(K_{n}, K_{e}\right) \in\left\{(0,0),\left(K_{n}, \infty\right),\left(\infty, K_{n}\right),(\infty, \infty)\right\}$. 


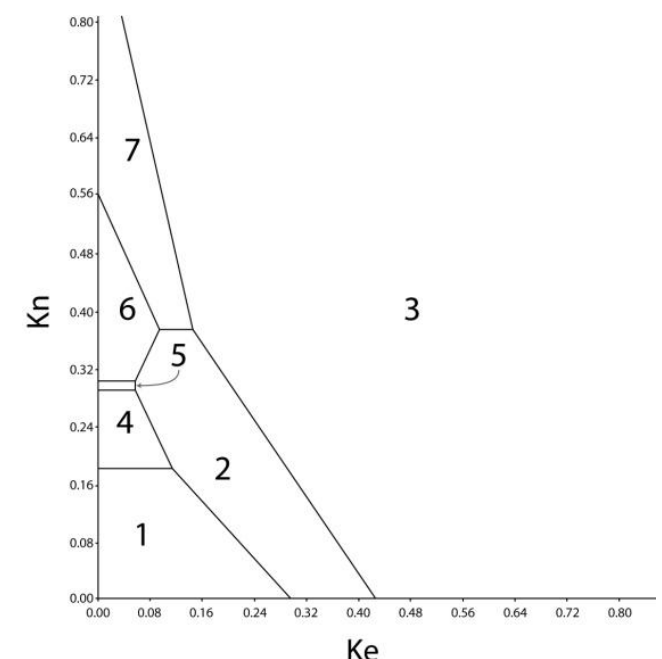

Figure 2.a: diagram of classes of cost.

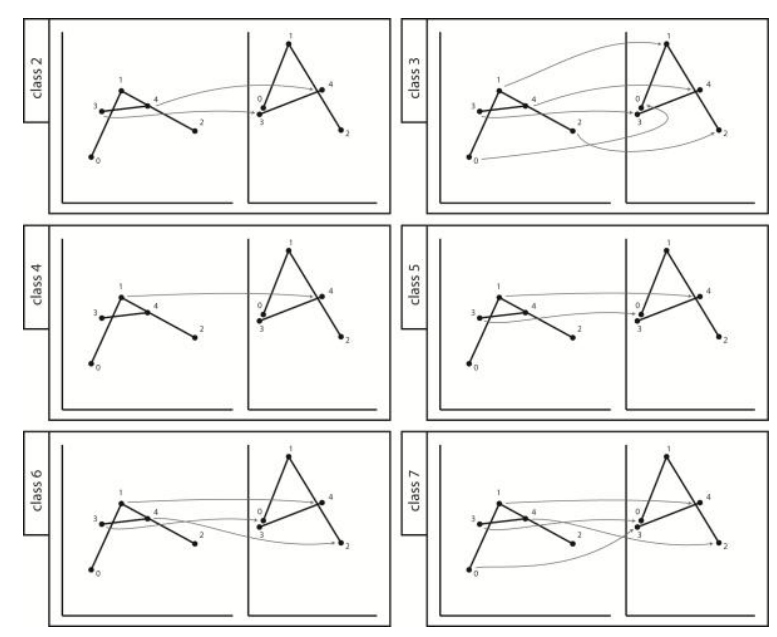

Figure 2.b: labellings related to of classes of cost.

Consider, as an example, graphs $G^{0}$ and $G^{10}$ of letter dataset [42] of class A. Fig. 2.a presents the classes of cost in the range of $K_{n}, K_{e} \in[0,0.8]$ for these graphs. The vertical axis corresponds to $K_{n}$ values and $K_{e}$ values are shown in the horizontal axis. In addition, Fig. 2.b shows the labellings that each class of cost produces.

We first analyze the labellings computed at $\left(K_{n}, K_{e}\right)=(0,0)$. At this special point every node insertion has a cost of zero, therefore, from the node point of view, the less costly assignation is to delete all nodes of the first graph and insert all nodes of the second graph. From the edge point of view, note that, if we assign all nodes to null the cost of edges will be eider substituted if the edge was not initially in the graph or deleted if the edge was initially on the graph; in both cases the edges cost will be zeros. Consequently, we can ensure that at point $\left(K_{n}, K_{e}\right)=(0,0)$, either because all nodes from both graphs will be assigned to null nodes of the other graph or because both graphs are isomorphic.

Analyzing Labellings attached to the vertical axis, that is $\left(K_{n}, K_{e}\right) \in(0 . . \infty, 0)$, it is clear that from high to low values of $K_{n}$ labellings associated with each class (Fig. 2.b) go from substituting all nodes $\left(\Omega_{3}^{0,10}\right)$ to only performing insertions and deletions $\left(\Omega_{1}^{0,10}\right)$. However, an interesting fact is that not all node substitutions are sub-contained in the adjacent label with lower $K_{n}$. We see that this happens in some classes $\Omega_{1}^{0,10} \subset \Omega_{4}^{0,10} \subset \Omega_{5}^{0,10} \subset \Omega_{6}^{0,10} \subset \Omega_{7}^{0,10}$ 
but not in others $\Omega_{3}^{0,10}$. Note that even the insertion and deletion of edges is not considered, edge substitution it is.

In the considered special case where $K_{e}=0$, notice that if edges do not have attributes, $C_{e s}(\cdot)=0$, the problem of computing the graph edit distance turns from the quadratic assignment problem to the linear assignment problem.

An interesting special value when moving over the $K_{n}$ axis is the value of $\left(K_{n}, K_{e}\right)=(\infty, 0)$. The labelling computed using this value maximizes assignation from nodes of the first graph to nodes of the second graph considering the minimum number of null assignations required which is $\left|\mathrm{R}^{\mathrm{p}}-\mathrm{R}^{\mathrm{q}}\right|$.

We now consider the labellings we obtain when moving over the $K_{e}$ axis. That is, we consider values $\left(K_{n}, K_{e}\right) \in(0,0 . . \infty)$. It is obvious that different values of $K_{e}$ force the result to be more structurally correct. However, forcing in addition $K_{n}=0$, it does not necessarily mean that the node attributes are not considered. In fact they are, due to node substitution cost is considered. In the example of Fig. 2 see that as we move $K_{e}$ towards $+\infty$, the classes change to force the labellings to be more structurally consistent. Note again how node substitutions are sometimes not sub-contained adjacent labels, e.g. $\Omega_{6}^{0,10} \subset \Omega_{7}^{0,10} \not \subset \Omega_{3}^{0,10}$.

In the extreme case $\left(K_{n}, K_{e}\right)=(0, \infty)$, we can affirm that the resulting optimal isomorphism, if enough null nodes are provided and edges do not have attributes, corresponds to the maximal common sub-graph as demonstrated in [30]. If we aim to obtain the maximal common sub-graph when attributes are present in edges, the edge substitution cost must restrict edges to have the same attribute and so the edge substitution cost must be defined as Table 1 row 1.

The final extreme value to analyze corresponds to $\left(K_{n}, K_{e}\right)=(\infty, \infty)$. In most of the cases while using these costs, the resulting labelling maximizes the node substitutions and edge substitutions at the same time. However, this double maximization can be troublesome in several cases. Considering this issue, we differentiate between two types of $\widehat{\Omega}$ (Def. 3) sets. 
The first corresponds to graphs where for values of $\left(K_{n}, K_{e}\right)=(\infty, 0)$ the optimal labelling is equivalent to the optimal labelling for values of $\left(K_{n}, K_{e}\right)=(0, \infty)$. That is, the structurally optimal and the semantically optimal labellings are equivalent. This is shown in Fig. 2.a. We consider this situation to be the desired case when applying graph matching to pattern recognition, because two optimally labelled similar objects should maximize structural and syntactical relations in the same labelling. The second type of labelling spaces corresponds to functions in which the optimal semantic labelling differs from optimal structural labelling. An example is shown in Fig. 3.a and 3.b which show graphs $G^{35}$ and $G^{72}$ of the letter dataset [42] of class $\mathrm{A}$. See that optimal labellings when $\left(K_{n}, K_{e}\right)=(\infty, 0)$ and $\left(K_{n}, K_{e}\right)=(0, \infty)$ differ. When this situation occurs in the application at hand, we must decide which labellings we prefer to optimize, structural or semantic.
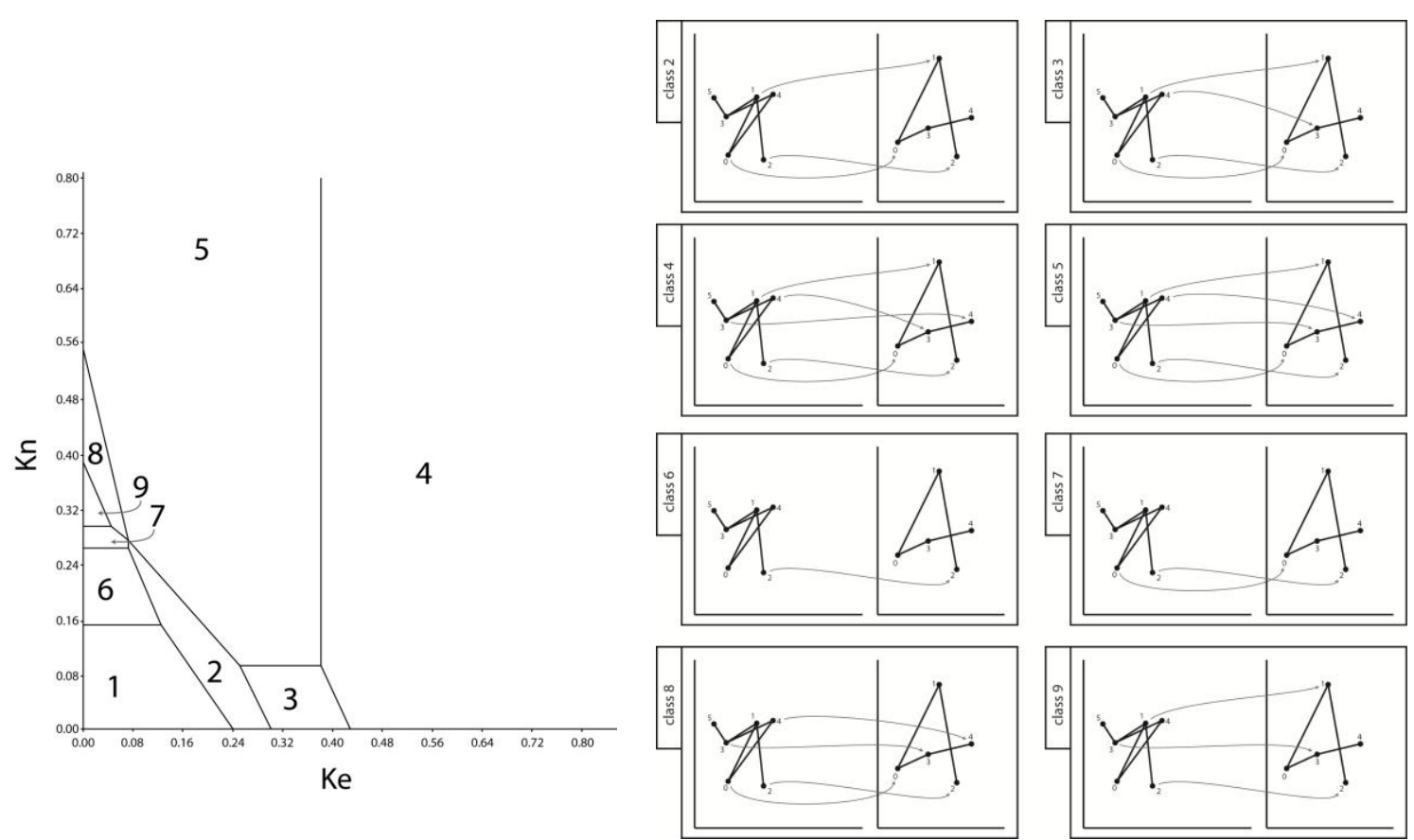

Figure 3.a: diagram of classes of cost. Figure 3.b: labellings related to of classes of cost.

Note that property 2 implies that the graph edit cost is not an injective function due to several labelling can give the same optimal cost. 


\subsection{Property of the Edit Surface}

Property 3. Given two graphs, $G^{p}$ and $G^{q}$, the function EditSurface ${ }_{G}{ }^{p} G_{G}^{q}$ monotonically increases. In other words, EditSurface ${ }_{G}{ }_{, G} q\left(p_{1}\right) \leq$ EditSurface $_{G^{p}, G} q\left(p_{2}\right)$ where $p_{1}=$ $\left(K_{n}^{1}, K_{e}^{1}\right), p_{2}=\left(K_{n}^{2}, K_{e}^{2}\right), K_{n}^{1} \leq K_{n}^{2}$ and $K_{e}^{1} \leq K_{e}^{2}$.

\section{Demonstration}

We know from Property 1 that the $\left(K_{n}, K_{e}\right)$ bi-dimensional space can be divided into several classes of cost, $\Omega\left(f_{1 . . m}\right)$. Each class of cost $\Omega\left(f_{i}\right)$ is represented by its plane equation (10). We know from Definition 1 that values $a_{f_{i}}, b_{f_{i}}$ and $c_{f_{i}}$ are positive. Thus, we can conclude that within each class of cost $\Omega\left(f_{i}\right)$, costs monotonically increase.

It is important to demonstrate that where two classes of cost intersect, costs do not decrease but remain equal or increase. Two labellings change their optimality when costs for both classes are equal, that is, when the equation (12) for two different labellings, $f$ and $f^{\prime}$, is equal. Using this min operator $(\leq)$, cost cannot decrease and so when two classes intersect, costs keep increasing $\square$
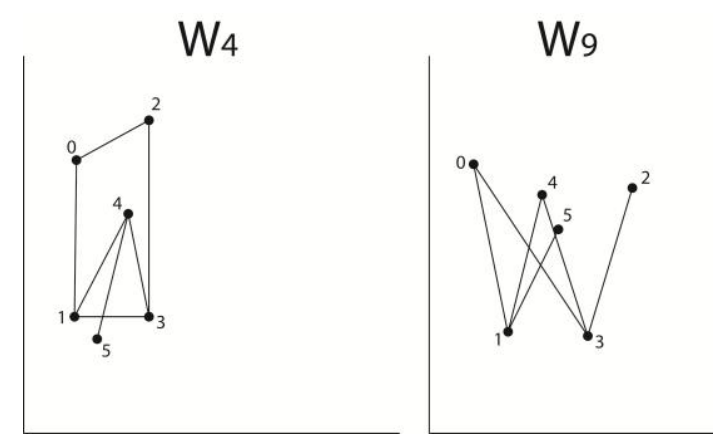

Figure 4.a: two graphs.

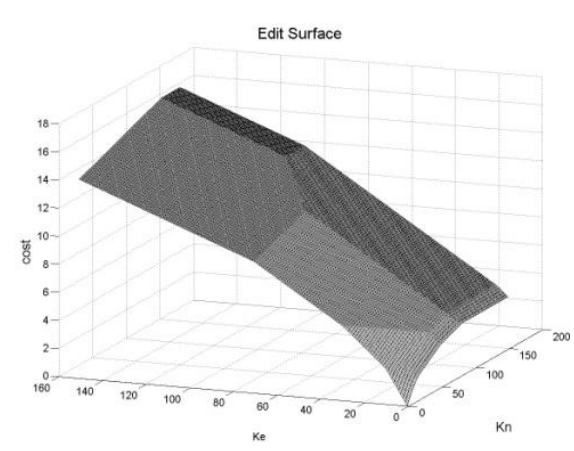

Figure 4.b: example of Edit Surface.

\section{Applications of the Class of Costs and Edit Surface}

In this section we illustrate how the given properties can aid some graph matching problems and describe three applications where the described properties can be used. 


\subsection{Interactive and Adaptive Graph Recognition}

The aim of the application we present is to learn a model that represents a set of graphs such that the labelling obtained by the graph matching algorithm is as similar as possible to the labelling between graph nodes imposed by a human expert. Part of the definitions, methods and algorithms commented here have been published in $[43,44]$.

In most of the applications, the labelling between nodes is only partially considered. This is because it is considered in the first stages of the pattern recognition process, in which it is desired to find a similarity measure between graphs. But, when this similarity value is obtained (the final distance value between graphs given the labelling), the knowledge of the labelling is not considered any more. Nevertheless, we consider that although the graph is properly classified or identified, the result of the comparison (the final distance value) has not sense if the labelling between their local parts is far from the labelling proposed by the human specialist.

We defined in [43] an interactive and adaptive graph recognition model with the aim of increasing the quality of the labelling between the graph to be identified and the reference graphs of the database. To that aim, we have extended the graph recognition model to consider the labelling between nodes proposed by a human specialist. This new knowledge is incorporated into the system and used to modify the weights of the model (such as $K_{n}$ and $K_{e}$ ) that tune the similarity function between graphs.

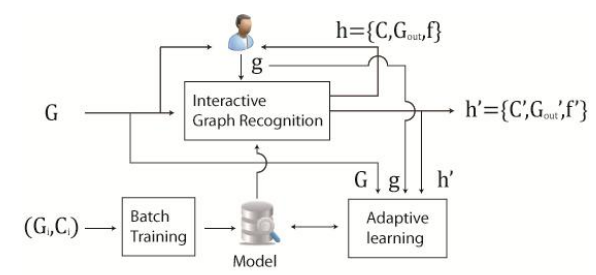

Figure 5. Scheme of the Interactive and Adaptive Graph Recognition Model.

The batch training process of our application, shown in Fig. 5, generates the first knowledge of the system that forms the model given a set of pairs (graph, class) and other parameters, such 
as $K_{n}$ and $K_{e}$. The Interactive Recognition process generates a first hypothesis $h=$ $\left\{C, G_{\text {out }}, f\right\}$ given an input graph $G$ and using the model. This hypothesis is composed by a class $C$, a graph with the minimum distance $G_{\text {out }}$ and a labelling $\mathrm{f}$ between both graphs. When the human proposes a new labelling $g$, the interactive process generates the final hypothesis $h^{\prime}$ using the model and also the human interaction $g$. Note that, $h^{\prime}$ can be completely different from $h$. Not only the graph and labelling can be different but also the class. Finally, the Adaptive Learning process updates the parameters in the model. Specifically, two of these parameters are $K_{n}$ and $K_{e}$. Computing the new values of these parameters is a process related to the aim of this paper since the new values are obtained through the labelling space. Moreover, the following algorithm needs property 1 to perform properly.

The inputs of the algorithm used to update $K_{n}$ and $K_{e}$ are the input graph $G$, the labelling imposed by the user $g$ and the final output graph $G_{\text {out }}^{\prime}$. The outputs of the algorithm are the new values of $K_{n}$ and $K_{e}$ which maximize the labellings proposed by the expert. The main idea of the algorithm is to build a histogram of the classes of costs that appeared while using the general model. That is, each time the algorithm receives a new input, the class of costs is obtained, $\Omega^{G, G_{\text {out }}^{\prime}}(\mathrm{g})$, and added to the histogram. Thus, the peak of the histogram (there could be several peaks) represents the values of $K_{n}$ and $K_{e}$ that maximize the similarity of the human labellings with the labellings proposed by the system.

Figure 6 (reprinted from [43]) shows the evolution of values of $K_{n}$ and $K_{e}$ when new graphs are added into the system. The system has been initialised by four different values of $K_{n}$ and $K_{e}$. The initial values are the most external points. We see that when the algorithm converges, the four experiments converge to the same final value of $K_{n}$ and $K_{e}$. 


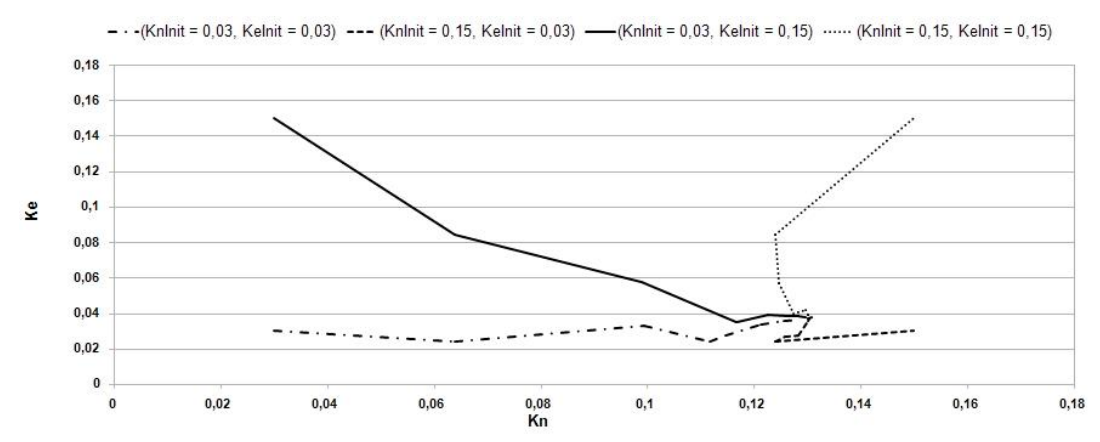

Figure 6. Evolution of $K_{n}$ and $K_{e}$ in four different initialisations.

\subsection{Analysis of the behaviour of human similarity measures}

Given a pair of images that represent objects (pictures, handwritten characters...), humans can decide if they are similar or not or even they can decide certain degree of similarity. This is because we have an inherent similarity function (difficult to be mathematically defined) that may be application dependent. When we aim to solve the problem of automatically describing this similarity through automatic structural pattern recognition, it is usual to convert these images to graphs and apply a distance measure between graphs.

Graph edit distance has some application dependent weights that can be manually tuned. Some research has been done to automatically obtain these weights such that the overall recognition ratio is maximised given a database $[33,34]$ or the difference between the node bijection between both graphs imposed by the specialist and the node bijection obtained by the machine is reduced [43]. If we have enough theoretical information to understand the behaviour about the graph distance at hand, it is possible to go a step further. It is possible to investigate if the inherent distance measure between nodes or between arcs that the user has, given an application, approaches the one that the method defines. Property 2 says that there could be two different labellings between nodes that are optimal at the same class of costs. These two labellings can be seen as different interpretation of the representation.

Suppose we want to compare pictures and we have extracted a region adjacency graph from each image. A region adjacency graph is a graph in which nodes represent important regions of the images. The attribute of the regions may be the average colour, the area, the circularity of 
the region and so on. There is a graph arc if regions are adjacent. There could be some attributes on the arcs such as the length of the border between regions.

Suppose we compute the cost class given the labelling imposed by a human specialist for all the graph comparisons.

Then, some different situations can happen.

The average value of $K_{n}$ of all the cost class is low given all the graph comparisons. In this case, the specialist believes the semantic information on the nodes is very important. Therefore, the specialist considers two images are similar if they have similar regions but independently of the position of these regions.

The average value of $K_{e}$ of all the cost class is low given all the graph comparisons. This case is the opposite of the last one. The user believes the most important aspect while comparing two images is the relation between regions (i.e. their relative position) although these regions seem to be very different (i.e. different area or colour)

The area of the union of the cost classes of all the comparisons is big although the intersection is small. This means that the specialist has different perceptions of the importance of the relations (arcs) respect the semantic information (nodes) depending on the images. This situation appears when the representation of the images does not include the feeling of the specialist.

An opposite case appears when the area of the union of the cost classes of all the comparisons is small. This case appears when the node bijections between both graphs that the user proposes are never the optimal ones or are only optimal in a small domain of $K_{n}$ and $K_{e}$. In the case that the general cost class is elongated through the $K_{e}$ axis, the inherent distance between image regions of the user performs in a different way than the distance between graph nodes of the system. On the contrary, in the case that the general cost class is elongated through the $K_{n}$ axis, the system captures in a different way the relations between these regions. 
In this way, by analyzing the application at hand and how the Graph Edit Distance behave over the graph representation and the data itself, we are able to adapt, change or replace the distance measure we are using.

\subsection{Reducing the distance error of sub-optimal graph matching algorithms}

Property 3 defines that the edit surface must increase when $K_{n}$ and $K_{e}$ increases. This certainly occurs when using optimal algorithms to compute the labellings given the values of $K_{n}$ and $K_{e}$. However, we cannot assume these properties hold true if we compute the labelling space using suboptimal algorithms. Two examples are shown in Fig. 7.a and 7.b, which show $\widehat{\Omega}$ obtained by the Graduated Assignment [45] using the graphs in Fig. 2.b and 3.b. We see that most of the regions computed by the Graduated Assignment are not convex. A good approach for improving sub-optimal algorithms that minimize Graph Edit Distance would be to modify them to hold for Property 1 while predicting the labelling given some certain point in the labelling space.

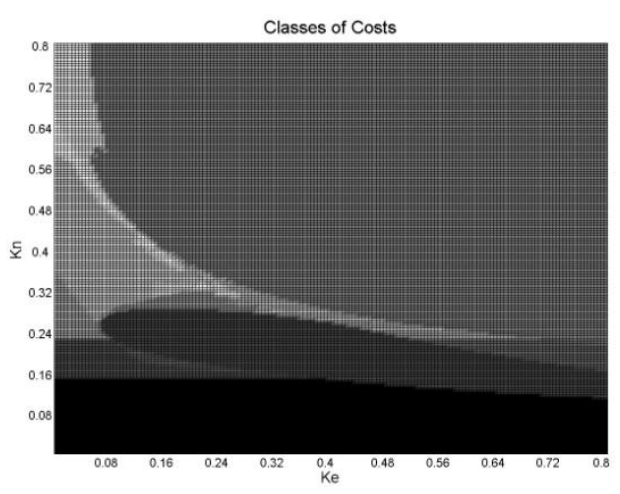

Figure 7.a: example of class of cost

computation using a suboptimal algorithm.

Graphs used are shown in Fig. 2.b.

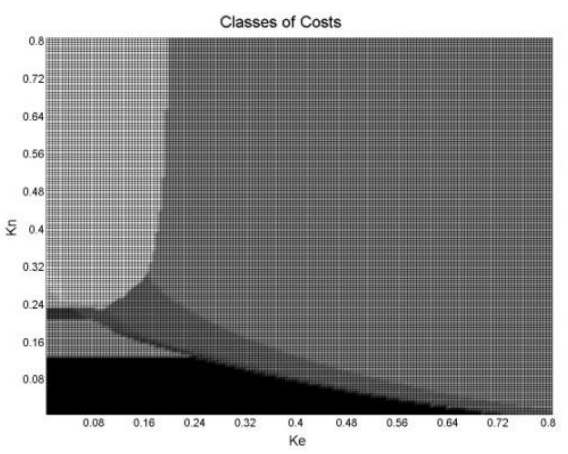

Figure 7.b: example of class of cost computation using a suboptimal algorithm. Graphs used are shown in Fig. 3.b.

Moreover, the application of Property 3 could help suboptimal algorithms to achieve better performance in graph distance computations. Fig. 8.a and 8.b show the EditSuface for a range of $K_{n}$ and $K_{e}$ values. Fig. 8.a is computed using the graphs of Fig. 2.b and Fig. 8.b using the 
graphs of Fig. 3.b. Both figures show two surfaces. The first, printed in solid gray scale, is computed using an optimal A* algorithm. The second, printed using transparency is computed using the Graduated Assignment [45] algorithm. We see how the Graduated Assignment does not provide a good approximation for high $K_{e}$ and relatively low $K_{n}$ values. This means that the graduated assignment is able to compute very good edge labellings but fail in computing node assignments. We see that Property 3 does not hold true for surfaces computed by the Graduated Assignment algorithm. A simple way of improving graph distance computations could be to compute several costs for different $K_{e}$ and $K_{n}$ values and average the results to force Property 3 to hold. We assume that the computational cost of computing few suboptimal algorithms is lower than one optimal algorithm.

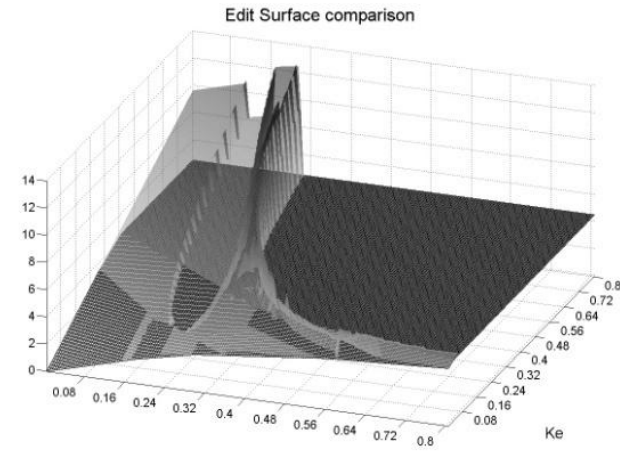

$\mathrm{kn}$

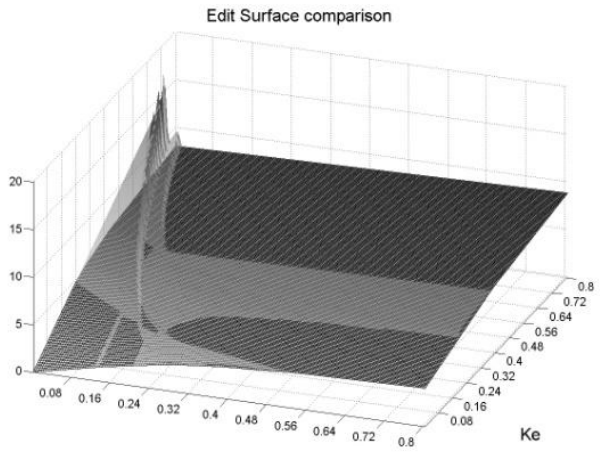

Kn
Figure 8.a: edit surfaces given by an optimal and a sub-optimal graph matching algorithm. Used graphs are shown in Fig 2.b.
Figure 8.b: edit surfaces given by an optimal and a sub-optimal graph matching algorithm. Used graphs are shown in Fig 3.b.

\section{Conclusions}

Most of the applications based on graphs algorithms rely mainly on a precise definition of a distance between two graphs. There are several definitions in use. However, the most used is probably the Graph Edit Distance or some variations of it. In this sense, a well known theoretical study of the distance is required. In this article, we presented some advances on the theoretical study of the Graph Edit Distance. We characterized the shape of the classes of 
costs presented previously in [30]. In addition, we described the shape of the Graph Edit Distance for the use of every possible graph edit constant. These advances are summarized in form of 3 properties which are adequately proven. To demonstrate the practical utility of the properties, we have presented three applications where these properties are used.

From the presented work, we can summarize several conclusions. We see by means of two examples that the number of labellings in the labelling space is relatively low with respect all possible labellings we should be able to obtain between two graphs. We see that variations of $K_{n}$ and $K_{e}$ constants do not give enough flexibility to learn a great amount of distinct isomorphism between two graphs. Different values of $K_{n}$ define how much we trust a possible assignation. However, this value is not locally tested but globally and so resignations of nodes are possible in consecutive labellings in the $K_{n}$ axis. $K_{e}$ define how structurally correct we desire the solution to be. The combination of these two constants allows computing some different labellings but the modification of node-to-node and edge-to-edge distances are required to provide to the Graph Edit Distance flexibility in learning every possible node-tonode bijection. Besides, the shape of the edit surface is clearly determined. This knowledge is important to evaluate the quality of suboptimal algorithms and in addition to aid in improving its results. 


\section{References}

1. Conte, D., et al., Thirty Years of Graph Matching In Pattern Recognition. International Journal of Pattern Recognition and Artificial Intelligence, 2004. 18(3): p. 265-299.

2. Xia, S. and E. Hancock, 3D Object Recognition Using Hyper-Graphs and Ranked Local Invariant Features, in Structural, Syntactic, and Statistical Pattern Recognition. 2008. p. 117-126.

3. He, L., C. Han, and W. Wee, Object recognition and recovery by skeleton graph matching, in International Conference on Multimedia \& Expo. 2006.

4. He, L., et al., Graph matching for object recognition and recovery. Pattern Recognition Letters, 2004. 37(7).

5. Neuhaus, M. and H. Bunke, Self-organizing maps for learning the edit costs in graph matching. Transactions on Systems, Man, and Cybernetics, 2005. 35(3): p. 305-314.

6. Riesen, K. and H. Bunke, Graph Classification Based on Vector Space Embedding. International Journal of Pattern Recognition and Artificial Intelligence, 2009. 23(6): p. 1053-1081.

7. Emms, D., R. Wilson, and E. Hancock, Graph matching using the interference of discrete-time quantum walks. Image and Vision Computing, 2009. 27(7): p. 985-1002.

8. Caetano, T., et al., Learning Graph Matching. Transaction on Pattern Analysis and Machine Intelligence, 2009. 31(6): p. 1048-1058.

9. Williams, M., R. Wilson, and E. Hancock, Multiple Graph Matching with Bayesian Inference. Pattern Recognition Letters, 1997. 18: p. 1275-1281.

10. Konc, J. and D. Janežič, A Branch and Bound Algorithm for Matching Protein Structures, in Adaptive and Natural Computing Algorithms. 2007. p. 399-406.

11. Sanromà, G., R. Alquézar, and F. Serratosa, Smooth Simultaneous Structural Graph Matching and Point-Set Registration, in Workshop on Graph-based Representations in Pattern Recognition. 2011. p. 142-151.

12. Romero, A. and M. Cazorla, Topological SLAM Using Omnidirectional Images: Merging Feature Detectors and Graph-Matching, in Advanced Concepts for Intelligent Vision Systems. 2010.

13. Thrun, S. and M. Montemerlo, The Graph SLAM Algorithm with Applications to Large-Scale Mapping of Urban Structures. International Journal of Robotics Research, 2006. 25(5-6): p. 403-429.

14. Ferrer, M., et al., Generalized median graph computation by means of graph embedding in vector spaces. Pattern Recognition Letters, 2010. 43(4): p. 1642-1615.

15. Xia, S. and E. Hancock, Learning Class Specific Graph Prototypes, in Image Analysis and Processing. 2009. p. 269-277.

16. Lozano, M.A. and F. Escolano, ACM attributed graph clustering for learning classes of images, in International conference on Graph based representations in pattern recognition. 2003.

17. Mukherjee, L., et al., Generalized median graphs and applications. JOURNAL OF COMBINATORIAL OPTIMIZATION, 2009. 17(1): p. 21-44.

18. Messmer, B. and H. Bunke, Fast error-correcting graph isomorphism based on model precompilation, in Image Analysis and Processing. 1997, Lecture Notes in Computer Science. p. 693-700.

19. Garey, M. and D. Johnson, Computers and Intractability: A Guide to the Theory of NPCompleteness. 1979.

20. Bunke, H. and K. Shearer, A graph distance metric based on the maximal common subgraph. Pattern Recognition Letters, 1998. 19(3-4): p. 255-259. 
21. Shapiro, L. and R. Haralick, A Metric for Comparing Relational Descriptions. Transactions on Pattern Analysis and Machine Intelligence, 1985. 7(1): p. 90-94.

22. Raveaux, R., J.-C. Burie, and J.-M. Ogier, A graph matching method and a graph matching distance based on subgraph assignments. Pattern Recognition Letters, 2010. 31(5): p. 394-406.

23. Sanfeliu, A. and K.-S. Fu, A Distance measure between attributed relational graphs for pattern recognition. IEEE transactions on systems, man, and cybernetics, 1983. 13(3): p. 353-362.

24. Gao, X., et al., A survey of graph edit distance. Pattern Analysis and applications, 2010. 13(1): p. 113-129.

25. Bunke, H. and G. Allermann, Inexact graph matching for structural pattern recognition Pattern Recognition Letters, 1983. 1(4): p. 245-253

26. Lladós, J., E. Martí, and J. Villanueva, Symbol Recognition by Error-Tolerant Subgraph Matching between Region Adjacency Graphs. TRANSACTIONS ON PATTERN ANALYSIS AND MACHINE INTELLIGENCE, 2001. 23(10): p. 1137-1143.

27. Justice, D. and A. Hero, A binary linear programming formulation of the graph edit distance. Transactions on Pattern Analysis and Machine Intelligence, 2006. 28(8): p. 1200-1214.

28. Riesen, K. and H. Bunke, Approximate graph edit distance computation by means of bipartite graph matching. Image and Vision Computing, 2009. 27(4): p. 950-959.

29. Riesen, K., M. Neuhaus, and H. Bunke, Bipartite Graph Matching for Computing the Edit Distance of Graphs, in GRAPH-BASED REPRESENTATIONS IN PATTERN RECOGNITION. 2007.

30. Bunke, H., Error Correcting Graph Matching: On the Influence of the Underlying Cost Function. Transactions on Pattern Analysis and Machine Intelligence, 1999. 21(9): p. 917-922.

31. Bunke, H., On a relation between graph edit distance and maximum common subgraph. Pattern Recognition Letters, 1998. 18(8): p. 689-694.

32. Rice, S., H. Bunke, and T. Nartker, Classes of cost functions for string edit distance. ALGORITHMICA, 1997. 18(2): p. 271-280.

33. Neuhaus, M. and H. Bunke, A Probabilistic Approach to Learning Costs for Graph Edit Distance, in International Conference on Pattern Recognition. 2007.

34. Neuhaus, M. and H. Bunke, Automatic learning of cost functions for graph edit distance. Information Sciences, 2006. 177(1): p. 239-247.

35. Kapsabelis, K., P. Dickinson, and K. Dogancay, Investigation of graph edit distance cost functions for detection of network anomalies, in Australian \& New Zealand Industrial and Applied Mathematics Journal. 2007.

36. Wong, A. and M. You, Entropy and Distance of Random Graphs with Application to Structural Pattern Recognition. Transaction on Pattern Analysis and Machine Intelligence, 1985. PAMI-7(5): p. 599-609.

37. Jain, A.K. and D. Maltoni, Handbook of Fingerprint Recognition. 2003, SpringerVerlag New York.

38. Serratosa, F., R. Alquézar, and A. Sanfeliu, Function-Described Graphs for modelling objects represented by attributed graphs. Pattern Recognition, 2003. 36(3): p. 781798.

39. Sanfeliu, A., F. Serratosa, and R. Alquézar, Second-Order Random Graphs for modelling sets of Attributed Graphs and their application to object learning and recognition. International Journal of Pattern Recognition and Artificial Intelligence, 2004. 18(3): p. 375-396.

40. Serratosa, F. and A. Sanfeliu, Signatures versus histograms: Definitions, distances and algorithms. Pattern Recognition Letters, 2006. 39(5): p. 921-934.

41. Grunbaum, B., Convex Polytopes. 2003. 
42. Riesen, K. and H. Bunke, IAM Graph Database Repository for Graph Based Pattern Recognition and Machine Learning, in Structural, Syntactic, and Statistical Pattern Recognition. 2008, Lecture Notes in Computer Science. p. 287-297.

43. Serratosa, F., A. Solé-Ribalta, and X. Cortés, Automatic Learning of Edit Costs Based on Interactive and Adaptive Graph Recognition, in Graph-Based Representations in Pattern Recognition. 2011, Lecture Notes in Computer Science. p. 152-162.

44. Solé-Ribalta, A. and F. Serratosa, Exploration of the Labelling Space Given Graph Edit Distance Costs in Graph-Based Representations in Pattern Recognition. 2011, Lecture Notes in Computer Science. p. 164-174.

45. Gold, S. and A. Rangarajan, A Graduated Assignment Algorithm for Graph Matching. Transaction on Pattern Analysis and Machine Intelligence, 1996. 18(4): p. 377-388.

\section{VITAE}

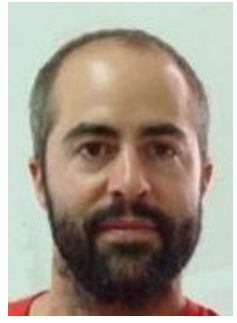

Albert Solé-Ribalta received the Computer Science Engineering degree and Master in Artificial Intelligence degree, from the Univeristat Rovira i Virgili (URV), in 2007. From 2005 to 2008 he was employed at URV as a collaborator professor. From 2006 to 2008, he worked at ITAKA where he was doing research in web based interfaces for multi-agent systems. On 2008, he received a pre-doctoral research grand from Universitat Rovira i Virgili. Since then, he is doing research in structural and syntactical pattern recognition based on graphs, at URV with collaboration with Univeristat Politècnica de Catalunya (UPC).

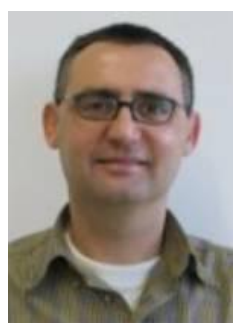

Francesc Serratosa was born in Barcelona, 24 may 1967. He received his Computer Science Engineering degree from the Universitat Politècnica de Catalunya (Barcelona) in 1993. Since then, he has been active in research in the areas of computer vision, robotics, structural pattern recognition and biometrics. He received his Ph.D. from the same university in 2000. He is currently associate professor of computer science at the Universitat Rovira $\mathrm{i}$ Virgili (Tarragona, Catalonia, Spain). He has published more than 50 papers and he is an active reviewer in some congresses and journals. He is the coordinator of the PhD course on Computer Science and Security at the Universitat Rovira i Virgili.

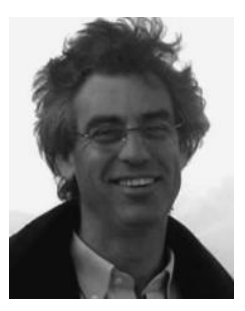

Alberto Sanfeliu received the B.S.E.E. and Ph.D. degrees from the Universitat Politéctica de Catalunya (UPC), Barcelona, Spain, in 1978 and 1982, respectively. In 1981, he joined the faculty of UPC and is currently a Full Professor of computational sciences and artificial intelligence. He is also the Director of the Institut de Robòtica i Informàtica Industrial, CSIC-UPC, Barcelona. He was the Coordinator of the European-Union-funded URUS Project. He has authored books in pattern recognition and SLAM, and published more than 200 papers in international journals and conference proceedings. He is the holder of several patents on quality control based on computer vision. He works on various theoretical aspects on pattern recognition, computer vision, and robotics and on applications on vision defect detection, tracking, object recognition, robot vision, and SLAM. 